\title{
AIGaInP optical source integrated with fiber links and silicon avalanche photo detectors in fiber optic systems
}

\author{
Mahmoud M. A. Eid', Shabana Urooj², Norah Muhammad Alwadai ${ }^{3}$, Ahmed Nabih Zaki Rashed ${ }^{4}$ \\ ${ }^{1}$ Department of Electrical Engineering, College of Engineering, Taif University, Kingdom of Saudi Arabia \\ ${ }^{2}$ Department of Electrical Engineering, College of Engineering, Princess Nourah bint Abdulrahman University, Riyadh, \\ Saudi Arabia \\ ${ }^{3}$ Department of Physics, College of Sciences, Princess Nourah bint Abdulrahman University, Riyadh, Saudi Arabia \\ ${ }^{4}$ Electronics and Electrical Communications Engineering Department, Faculty of Electronic Engineering, \\ Meniufia University, Egypt
}

\section{Article Info}

Article history:

Received Mar 3, 2021

Revised Jul 7, 2021

Accepted Jul 13, 2021

\section{Keywords:}

AlGaInP laser diode

Fiber link

Si APD

Integrated optics

\section{Corresponding Author:}

Shabana Urooj

Princess Nourah bint Abdulrahman University

Saudi Arabia

Email: SMUrooj@pnu.edu.sa

\begin{abstract}
This study has clarified aluminium gallium indium phosphide (AlGaInP) optical source integrated with fiber links and silicon avalanche photodetectors in fiber optic systems. The output spectral power, rise time, signal frequency and resonance frequency for AlGaInP laser diode. The laser diode rise time, output spectral power and resonance/signal frequencies versus injection current and ambient temperatures are sketched. The silica doped germanium fiber link pulse broadening and the signal fiber bandwidth are investigated against temperature variations. The signal per noise ratio is related to $\mathrm{Q}$ value and bit error rate (BER) at the receiving point $(\mathrm{Si}$ avalanche photodetector (APD)) are sketched with temperature.
\end{abstract}

This is an open access article under the CC BY-SA license.

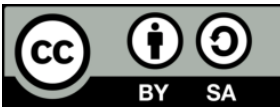

\section{INTRODUCTION}

The resulting signals become unrecognizable as individual information bits and the accurate detection of the original data signals become difficult at the receiver end which demodulates the optical signals into their original transmitted pulses [1]-[7]. The optical amplifiers are then required in very long transmission links to boost and amplify the weak and distorted optical signals for accurate retrieving of original signal in the receiver stage [8]-[15]. At the receiver end, the optical signals are detected and converted back into electrical signals by a light sensitive device known as a light detector or a photodetector (PD) like avalanche photodetector (APD) [16]-[23]

The optical transmitter unit is a transducer. It is composed of optical source and the driver circuit [24]-[27]. It converts the electrical input signals into corresponding light signals and launches them into the optical fiber. The light emitting diodes (LEDs) and laser diodes (LDs) are solid state semiconductor devices [28]-[33]. They are fabricated from different semiconductor materials which enable the designer to determine the emission wavelength. The gallium aluminum arsenide (GaAlAs) laser can emit the wavelengths range between 0.8 to $0.9 \mu \mathrm{m}$ while the indium gallium arsenide phosphide (InGaAsP) laser can emit the wavelength range between 1.0 and 1.6 $\mu \mathrm{m}$. Both LEDs and LDs can be modulated directly [34]-[39].

They enhance the optical communication systems and networks. The performance enhancement has been achieved through three directions of methodologies for thesis researches. The first methodology has been performed through performance considerations evaluation for different vertical cavity surface emitting 
lasers (VCSEL) structures and various electro-optic modulators types under influences of different spectral and thermal changes to determine the more effective VCSEL structure and better electro-optic modulator type [40]-[49].

This work has concentrated on AlGaInP optical source integrated with fiber links and silicon avalanche photodetectors in fiber optic systems. The output spectral power, rise time, signal frequency and resonance frequency for aluminium gallium indium phosphide (AlGaInP) laser diode. The laser diode rise time, output spectral power and resonance/signal frequencies versus injection current and ambient temperatures are sketched. The silica doped germanium fiber link pulse broadening and the signal fiber bandwidth are investigated against temperature variations. The signal per noise ratio is related to $\mathrm{Q}$ value and BER at the receiving point.

\section{CIRCUIT DESCRIPTION AND RESEARCH METHOD}

The output power $\left(\mathrm{P}_{0}\right)$ and applied bias voltage voltage for the laser diodes are given by [8]-[19]:

$$
\begin{aligned}
& P_{0}=22.65-3.65 V+1.543 V^{2}-0.07654 V^{3} \\
& V=2.18755+0.113597 I-0.003626 I^{2}+0.0004816 I^{3}
\end{aligned}
$$

With I is the injection current which is modeled by a function of ambiemnt temperature (K) as [8], [20]-[28]:

$$
I=a_{0}+a_{1} T+a_{2} T^{2}+a_{3} T^{3}
$$

Where for AlGaInP laser diode, the values are $\mathrm{a}_{0}=0.4321, \mathrm{a}_{1}=10.54 \times 10^{-4}, \mathrm{a}_{2}=1.324 \times 10^{-9}$, and $\mathrm{a}_{3}=0.8764 \times 10^{8}$. Where the laser diode bandwidth $\left(\mathrm{f}_{3-\mathrm{dB}}\right)$, its rise time $(\tau)$, and its resonance frequency $\left(\mathrm{f}_{\mathrm{r}}\right)$, are cast by the mathematical formulas [10,11-22]:

$$
\begin{aligned}
& f 3-d B=b_{0}+b_{1} I+b_{2} I^{2} \\
& \tau=c_{0}+c_{1} I+c_{2} I^{2} \\
& f r=d_{0}+d_{1} I+d_{2} I^{2}
\end{aligned}
$$

All the basic coefficients $b_{0}, b_{1}, b_{2}, c_{0}, c_{1}, c_{2}$, and $d_{0}, d_{1}, d_{2}$, of $f_{3-d B}, \tau$ and $f_{r}$ versus temperature $T$ for AlGaInP laser diode are listed in the series of equations as the following [10], [23]-[33]:

$$
\begin{aligned}
& b_{0}=1.43-0.976 T-0.08765 T^{2} \\
& b_{1}=1.546-0.0876 T-0.7654 T^{2} \\
& b_{2}=1.59-0.0876 T+0.07636 T^{2} \\
& c_{0}=0.87-3.685 T-0.0654 T^{2} \\
& c_{1}=-3.65+1.321 T-1.654 T^{2} \\
& c_{2}=33.65-3.165 T+0.0543 T^{2} \\
& d_{0}=2.987-0.765 T-0.7659 T^{2} \\
& d_{1}=00655-1.076 T+3.765 T^{2} \\
& d_{2}=6.543+0.327 T-0.43298 T^{2}
\end{aligned}
$$

For the silica doped germanium with fiber link refractive index can be modeled by the following formula [8], [9], [30]-[39]:

$$
n=\sqrt{\frac{h_{1} \lambda^{2}}{\lambda^{2}-h_{2}^{2}}+\frac{h_{3} \lambda^{2}}{\lambda^{2}-h_{4}^{2}}+\frac{h_{5} \lambda^{2}}{\lambda^{2}-h_{6}^{2}}}
$$

Where all the basic coefficients for the fiber link are cast as the following formulas:

$$
\begin{aligned}
\mathrm{h}_{1} & =0.8765\left(\frac{\mathrm{T}}{\mathrm{T}_{0}}\right)\left(\frac{\mathrm{x}}{0.054}\right) \\
\mathrm{h}_{2} & =1.2323\left(\frac{\mathrm{T}}{\mathrm{T}_{0}}\right)^{2} \\
h_{3} & =5.654\left(\frac{T}{T_{0}}\right)\left(\frac{x^{2}}{3.539}\right)
\end{aligned}
$$




$$
\begin{aligned}
\mathrm{h}_{1} & =1.986 \times 10^{4}\left(\frac{\mathrm{T}}{\mathrm{T}_{0}}\right)\left(\frac{1.54}{2.2 x}\right) \\
\mathrm{h}_{2} & =0.0432\left(\frac{\mathrm{T}}{\mathrm{T}_{0}}\right)^{2} \\
h_{3} & =0.00654\left(\frac{T}{T_{0}}\right)\left(\frac{x^{3}}{7.876}\right)
\end{aligned}
$$

Where $\mathrm{T}_{0}$ is the room temperature and $\mathrm{x}$ the percentage dopant ratio from germanium added to silica fiber link. The soliton transmission techniques based on the soliton peak power with the following expression [10]-[14], [20]-[29]:

$$
P_{1}=\frac{\Delta \lambda^{3} D_{t} A_{e f f}}{4 \pi^{2} c n_{2} \Delta \tau^{2}}
$$

Where $\mathrm{n}_{2}$ is the nonlinear coefficient in $\mathrm{m}^{2} /$ Watt, $\mathrm{D}_{\mathrm{t}}$ is total dispersion factor, $A_{\mathrm{eff}}$ is the cross section effective area for optic fiber link, and then by using (13), the total pulse broadening, and total signal bandwidth are estimated by [9], [10], [12]-[20]:

$$
\begin{aligned}
& \Delta \tau=\sqrt{\frac{\Delta \lambda^{3} D_{t} A_{e f f}}{4 \pi^{2} P_{1} n_{2} c}} \\
& B W_{\text {Sig. }}=\frac{0.44}{\Delta \tau L}
\end{aligned}
$$

The sig./noise ratio with the signal quality and BER at the receiving point expressed by [9]-[11], [13]-[20]:

$$
\begin{aligned}
& S N R=\frac{\left(G P_{R} \rho\right)^{2} R_{L}}{4 k T B \cdot W_{\text {sig. }}+2 e R_{L} B \cdot W_{\text {sig. }} G^{n}\left(I_{d}+\rho P_{R}\right)}, \\
& (S N R)_{d B}=10 \log S N R, \\
& B E R=0.5[1-\operatorname{erf}(0.345 S N R)]
\end{aligned}
$$

\section{DISCUSSION}

We have analyzed the quadrature semiconductor light source integrated with silica doped germanium fiber link in the complete matching silicon avalanche photodetectors. The output spectral power, resonance frequency, laser diode frequency bandwidth and laser diode rise time are analyzed and discussed. As well as the total pulse broadening, fiber signal bandwidth is also analyzed by using a Soliton transmission scheme-based fiber communication links. The sig./noise ratio and BER are investigated through the silicon avalanche photodetector or optical receiver side. All the obtained results are depending on the following parameters such as Ambient temperature $300 \leq \mathrm{T}, \mathrm{K} \leq 425, \mathrm{P}_{1}=100 \mathrm{~mW}$, photodetector responsivity $\rho=0.9$ A/W, $I_{d}=10 \mathrm{nA}$, Fiber link=10 km, $R_{L}=100 \mathrm{~K} \Omega, G=2$ for photodetector, $\lambda=1550 \mathrm{~nm}$, germanium doped ratio (x), $0.1 \leq \mathrm{x} \leq 0.3$, and Injection current (I), $5 \leq \mathrm{I}, \mathrm{mA} \leq 20$. As shown in Figure 1, the output spectral power variations with respect to laser injection current (LIC) at various ambient temperatures. The obtained result is assured that the increase of the injection current, results in the increase of the output spectral power of the laser. The output power at both the LIC of $5 \mathrm{~mA}$ and $300 \mathrm{~K}$ is $10 \mathrm{~mW}$. The output power at both the LIC of $20 \mathrm{~mA}$ and $300 \mathrm{~K}$ is $45 \mathrm{~mW}$. Figure 2 clarifies the laser signal frequency variations versus LIC variations at various ambient temperatures. The laser signal frequency increases with injection current increases. The laser signal frequency at both the LIC of $5 \mathrm{~mA}$ and $300 \mathrm{~K}$ is $12 \mathrm{GHz}$. The laser signal frequency at both the LIC of $20 \mathrm{~mA}$ and $300 \mathrm{~K}$ is $77 \mathrm{GHz}$. The higher the injection laser current the higher laser signal frequency.

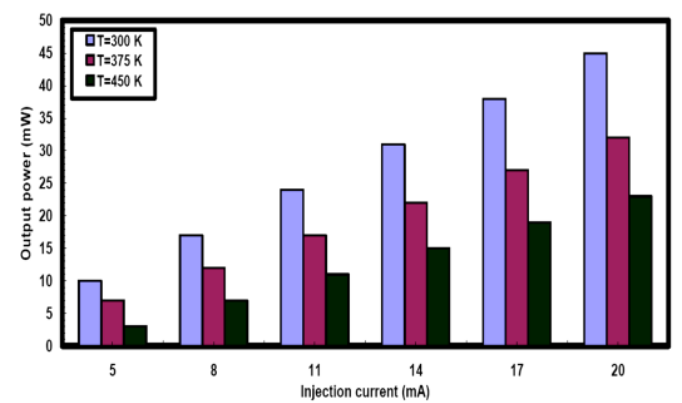

Figure 1. Output spectral power variations with respect to LIC at various temperatures 


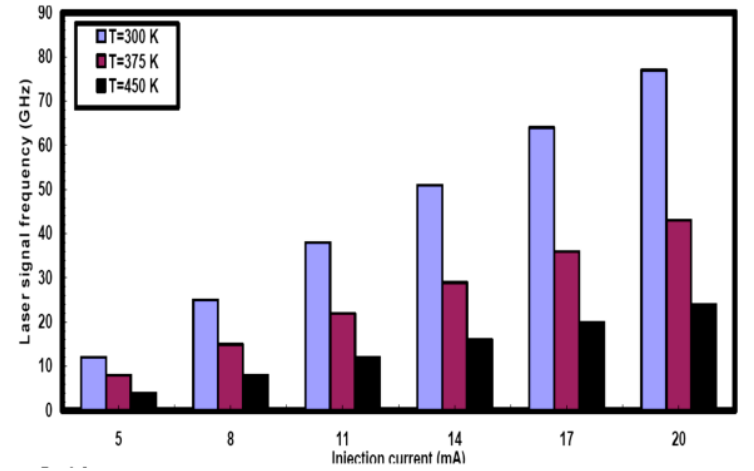

Figure 2. Laser signal frequency variations versus LIC variations at various temperatures

Figure 3 illustrates the laser rise time variations versus LIC variations at various temperatures. The laser rise time at both the laser current of $5 \mathrm{~mA}$ and $300 \mathrm{~K}$ is $10 \mathrm{~ns}$. The laser rise time increases with the increase of the injection current. The laser rise time at both the LIC of $20 \mathrm{~mA}$ and $300 \mathrm{~K}$ is $320 \mathrm{~ns}$. The higher the LIC the higher the laser rise time.

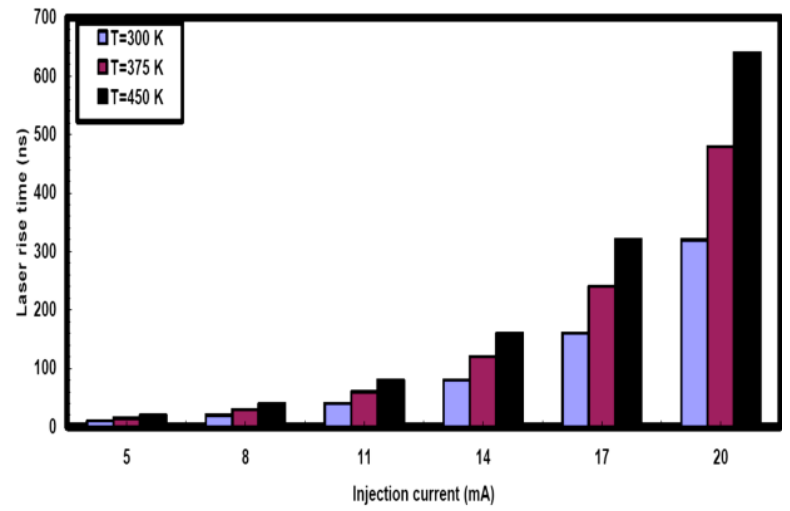

Figure 3. Laser rise time variations versus LIC variations at various temperatures

Figure 4 shows the laser resonance frequency variations versus LIC variations at various ambient temperatures. The laser resonance frequency increases with the increase of the injection current. The laser resonance frequency at both the LIC of $5 \mathrm{~mA}$ and $300 \mathrm{~K}$ is $25 \mathrm{GHz}$. The laser resonance frequency at both the LIC of $20 \mathrm{~mA}$ and $300 \mathrm{~K}$ is $60 \mathrm{GHz}$. The higher the LIC the higher laser resonance frequency. Table 1 summarizes the results of Figures 1, 2, 3, and 4 for the laser performance parameters under min/max injection current variations at various temperatures.

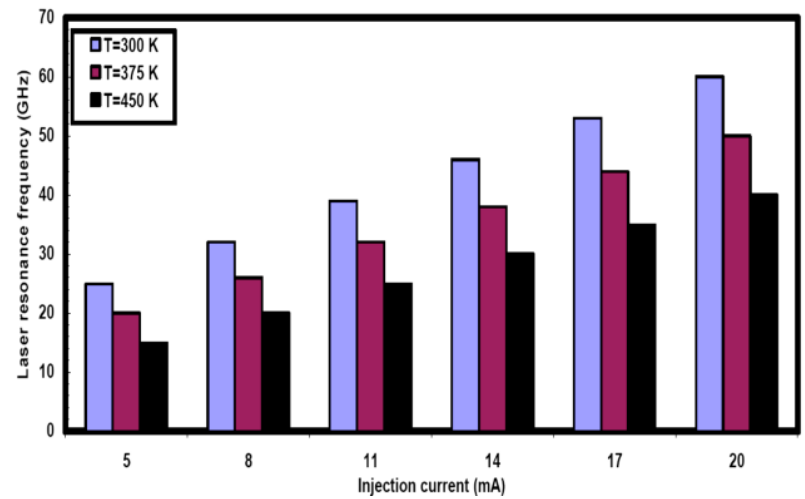

Figure 4. Laser resonance frequency variations versus LIC variations at various ambient temperatures 
Table 1. Laser performance parameters under $\min / \max$ injection current variations at various temperatures

\begin{tabular}{lcccccc}
\hline Laser performance parameters & \multicolumn{3}{c}{$5 \mathrm{~mA}$ injection current } & \multicolumn{3}{c}{$20 \mathrm{~mA}$ injection current } \\
& $300 \mathrm{~K}$ & $375 \mathrm{~K}$ & $450 \mathrm{~K}$ & $300 \mathrm{~K}$ & $375 \mathrm{~K}$ & $450 \mathrm{~K}$ \\
\hline Output power $(\mathrm{mW})$ & 10 & 7 & 3 & 45 & 32 & 23 \\
Laser frequency $(\mathrm{GHz})$ & 12 & 8 & 4 & 77 & 43 & 24 \\
Laser rise time (ns) & 10 & 15 & 20 & 320 & 480 & 640 \\
Laser resonance frequency $(\mathrm{GHz})$ & 25 & 20 & 15 & 60 & 50 & 40 \\
\hline
\end{tabular}

Figure 5 clarifies the fiber channel pulse broadening in relation to ambient temperature at different germanium dopant ratios. The fiber pulse broadening decreases with increasing the germanium dopant ratio and adjust the temperature at room temperature. The fiber channel pulse broadening at both the germanium dopant ratio of 0.1 and a room temperature of $300 \mathrm{~K}$ is $320 \mathrm{~ns}$. The fiber channel pulse broadening at both the germanium dopant ratio of 0.1 and an ambient temperature of $450 \mathrm{~K}$ is $500 \mathrm{~ns}$. The higher the dopant ratio in the fiber the lower the fiber pulse broadening. Figure 6 shows the fiber channel bandwidth in relation to ambient temperature at different germanium dopant ratios. The fiber channel bandwidth increases with the increase of the germanium dopant ratio and adjusts the temperature at room temperature. The fiber channel bandwidth at both the germanium dopant ratio of 0.1 and a room temperature of $300 \mathrm{~K}$ is $40 \mathrm{GHz}$. The fiber channel bandwidth at both the germanium dopant ratio of 0.1 and an ambient temperature of $450 \mathrm{~K}$ is 22 GHz. The higher the dopant ratio in the fiber the higher the fiber bandwidth.

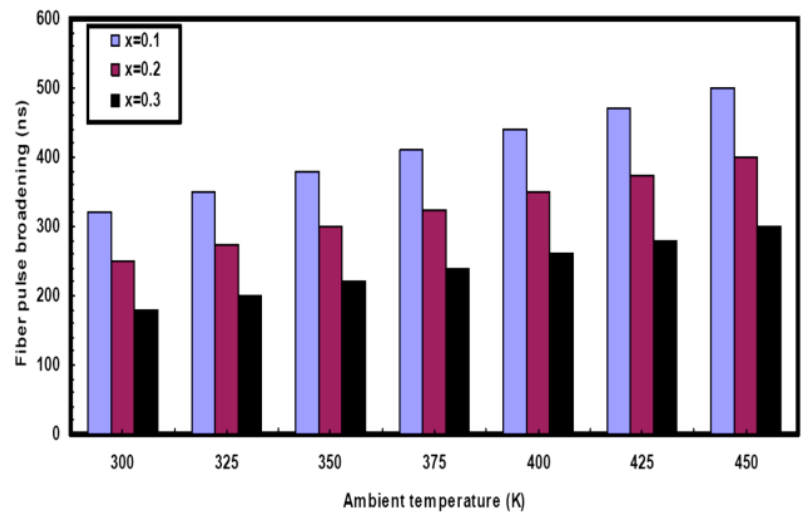

Figure 5. Fiber channel pulse broadening with temperature at different germanium dopant ratios

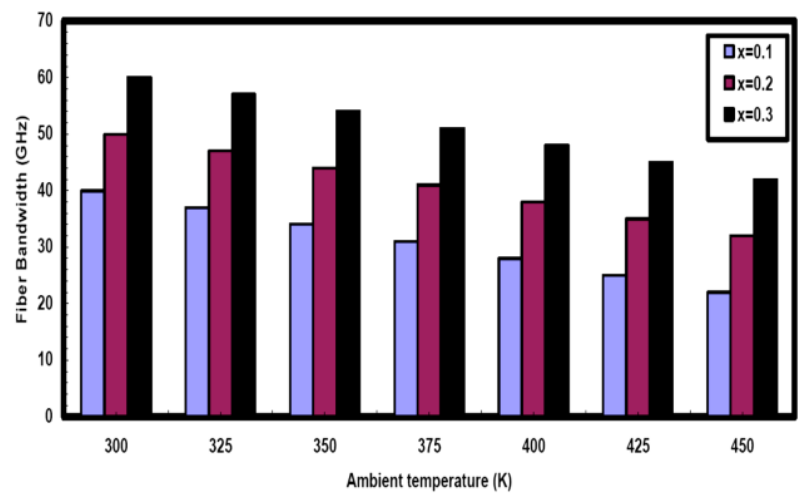

Figure 6. Fiber channel bandwidth with temperature at different germanium dopant ratios

Figure 7 clarifies the signal per noise ratio with the temperature at different germanium dopant ratios based optical receivers. The signal per noise ratio increases with the adjust the temperature at $300 \mathrm{~K}$ and the germanium dopant ratio at 0.3 . The sig./noise ratio at both the germanium dopant ratio of 0.1 and a room temperature of $300 \mathrm{~K}$ is $30 \mathrm{~dB}$. The signal per noise ratio at both the germanium dopant ratio of 0.1 and an ambient temperature of $450 \mathrm{~K}$ is $12 \mathrm{~dB}$. The higher the dopant ratio in the fiber the lower the sig./noise ratio. Figure 8 shows the BER against ambient temperature at different germanium dopant ratios based optical receivers. The data error rates decrease with the adjust the temperature at $300 \mathrm{~K}$ and the germanium dopant ratio at 0.3. The higher the dopant ratio in the fiber the higher the BER at the receiver side. Table 2 
summarizes the results of Figures 5, 6, 7 and 8 for the Table 2 Fiber/Photodetector performance parameters under min./max temperature variations at various germanium dopant ratios.

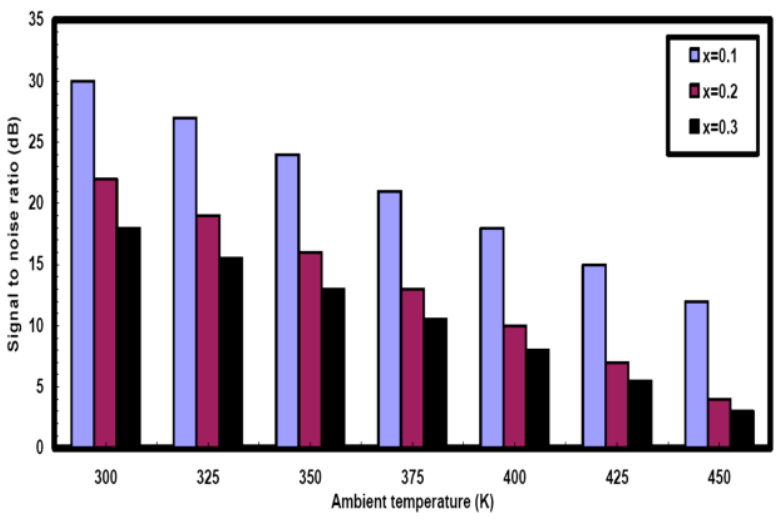

Figure 7. Signal per noise ratio variations with the temperature at various germanium dopant ratios based optical receivers

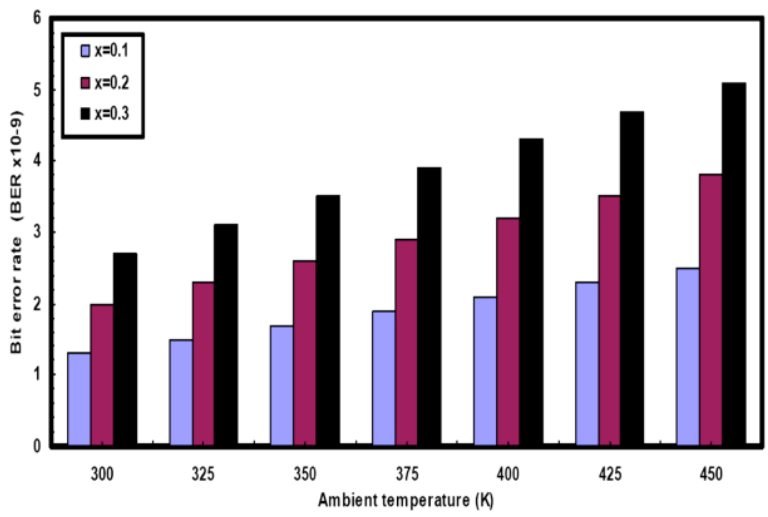

Figure 8. Data rate against temperature at various germanium dopant ratios based optical receivers

Table 2. Fiber/Photodetector performance parameters under min./max temperature variations at various germanium dopant ratios

\begin{tabular}{lcccccc}
\hline $\begin{array}{c}\text { Fiber/Photodetector performance } \\
\text { parameters }\end{array}$ & \multicolumn{2}{c}{$300 \mathrm{~K}$ room temperature } & \multicolumn{2}{c}{$450 \mathrm{~K}$ ambient temperature } \\
& $\begin{array}{c}10 \% \\
\text { dopant }\end{array}$ & $\begin{array}{c}20 \% \\
\text { dopant }\end{array}$ & $\begin{array}{c}30 \% \\
\text { dopant }\end{array}$ & $\begin{array}{c}10 \% \\
\text { dopant }\end{array}$ & $\begin{array}{c}20 \% \\
\text { dopant }\end{array}$ & $\begin{array}{c}30 \% \\
\text { dopant }\end{array}$ \\
\hline Fiber pulse broadening $(\mathrm{ns})$ & 320 & 250 & 180 & 500 & 400 & 300 \\
Fiber bandwidth $(\mathrm{GHz})$ & 40 & 50 & 60 & 22 & 32 & 42 \\
Signal to noise ratio at receiver $(\mathrm{dB})$ & 30 & 22 & 18 & 12 & 4 & 3 \\
Bit error rate $\left(\mathrm{BER} \times 10^{-9}\right)$ & 1.3 & 2 & 2.7 & 2.5 & 3.8 & 5.1 \\
\hline
\end{tabular}

\section{CONCLUSION}

AlGaInP laser diode source integrated with silica doped germanium fiber link and Si APD optical receivers are investigated in detail. The laser diode output spectral power, laser rise time, laser resonance frequency, laser signal bandwidth is sketched versus both injection current and ambient temperatures. The silica doped fiber link bandwidth and fiber link pulse width broadening are analyzed against temperature variations. The sig./noise ratio and data error rates at the receiver point are investigated against temperature variations. The obtained results are enhanced with room temperature and average injection current value of $10 \mathrm{~mA}$. The fiber link performance is upgraded with the increase dopant ratio of germanium up to $30 \%$ added to the silica fiber link. 


\section{ACKNOWLEDGMENT}

This research was funded by the Deanship of Scientific Research at Princess Nourah bint Abdulrahman University through the Fast-track Research Funding Program.

\section{REFERENCES}

[1] D. A. Vanderwater, I. H. Tan, G. E. Hofler, D. C. Defevere, and F. A. Kish., "High-brightness AlGaInP light emitting diodes," Proceedings of the IEEE, vol. 85, no. 11, 1997, pp. 1752-1764, doi: 10.1109/5.649654.

[2] W. C. Chong, K. M. Wong, Z. J. Liu, and K. M. Lau, "60.4: A Novel Full-Color 3LED Projection System using RG-B Light Emitting Diodes on Silicon (LEDoS) Micro-displays," SID Symposium Digest of Technical Papers, vol. 44, no. 1, pp. 838-841, 2013, doi: 10.1002/j.2168-0159.2013.tb06348.x.

[3] S. F. Wan, C. Y. Ho, Y. G. Chen, Q. B. Li, and F. F. Yao, "Analysis of Junction Temperature of AlGaInP LED," Key Engineering Materials, vol. 777, pp. 138-142, 2018, doi: 10.4028/www.scientific.net/KEM.777.138.

[4] F. Gou, et al., "Angular color shift of micro-LED displays," Optics Express, vol. 27, no. 12, pp. A746-4757, 2019, doi: 10.1364/OE.27.00A746.

[5] Y. Lee, C. Lee, and C. Chen, "Effect of Surface Texture and Backside Patterned Reflector on the AlGaInP LightEmitting Diode: High Extraction of Waveguided Light," IEEE Journal of Quantum Electronics, vol. 47, no. 5, pp. 636-641, 2011, doi: 10.1109/JQE.2011.2107891

[6] Y. Wang, et al., "High-performance AlGaInP light-emitting diodes integrated on silicon through a superior quality germanium-on-insulator," Photonics Research, vol. 6, no. 4, pp. 290-295, 2018, doi: 10.1364/PRJ.6.000290.

[7] C.-M. Kang, et al., "Monolithic integration of AlGaInP-based red and InGaN-based green LEDs via adhesive bonding for multicolor emission," Scie. Reports, vol. 7, no. 1, pp. 1-9, 2017, doi: 10.1038/s41598-017-11239-4.

[8] N. C. Helman, J. E. Roth, D. P. Bour, H. Altug, and D. A. Miller, "Misalignment-Tolerant Surface-Normal LowVoltage Modulator for Optical Interconnects," IEEE Journal of Selected Topics in Quantum Electronics, vol. 11, no. 2, pp. 338-342, 2005, doi: 10.1109/JSTQE.2005.845613.

[9] A. M. Abd El-Naser, N. Ayad, A. N. Z. Rashed, and M. H. Hazem, "Soliton Transmission Capacity of Vertical Cavity Surface Emitting Lasers (VCSELs) Degradation under Thermal Irradiated Fields," International Journal of Multidisciplinary Sciences and Engineering (IJMSE), vol. 2, no. 3, pp. 20-30, June 2011.

[10] A. V. Krishnamoorthy, et al., "Computer Systems Based on Silicon Photonic Interconnects," Proceedings of the IEEE, vol. 97, no. 7, 2009, pp. 1337-1361.

[11] Y. C. Chang, C. S. Wang, and L. A. Coldren, "High Efficiency, High Speed VCSELs with 35 Gbit/s Error Free Operation," Electron. Lett., vol. 43, no. 19, pp. 1022-1023, 2007.

[12] J. A. Timpson, et al., "Single Photon Sources Based upon Single Quantum Dots in Semiconductor Microcavity Pillars," Journal of Modern Optics, vol. 54, no. 2-3, pp. 453-465, 2007, doi: 10.1080/09500340600785055

[13] I. O'Connor, et al., "Systematic simulation-based predictive synthesis of integrated optical interconnect," IEEE Transactions on Very Large Scale Integration (VlSI) Systems, vol. 15, no. 8, pp. 927-940, Aug. 2007.

[14] M. Gnan, S. Thoms, D. S. Macintyre, R. M. De La Rue, and M. Sorel, "Fabrication of Low Loss Photonic Wires in Silicon-On-Insulator Using Hydrogen Silsesquioxane Electron Beam Resist," Electronics Letters, vol. 44, no. 2 , pp. 115-116, 2008, doi: 10.1049/el:20082985.

[15] M. Asada, Y. Miyamoto, and Y. Suematsu, "Gain and the Threshold of Three-Dimensional Quantum-Box Lasers," IEEE Journal of Quantum Electronics, vol. 22, no. 9, 1986, pp. 1915-1921. doi:10.1109/JQE.1986.1073149.

[16] N. N. Ledentsov, et al., "Optical Properties of Heterostructures with InGaAs- GaAs Quantum Clusters," Semiconductors, vol. 28, no. 8, pp. 832-834, 1994.

[17] U. Brinkmann, "Nonlinear optics-Spatial solitons of moderate power interact," Laser Focus World, vol. 40, no. 4, pp. 32-48, 2004.

[18] I. Maimistov, "Solitons in nonlinear optics," Quantum Electron., vol. 40, no. 9, pp. 756-781, 2010, doi: 10.1070/QE2010v040n09ABEH014396.

[19] X. G. Lin, W. J. Liu, and M. Lei, "Oscillating solitons in nonlinear optics," Pramana, vol. 86, no. 3, pp. 575-580, 2016, doi: 10.1007/s12043-015-1020-x.

[20] Y. Song, X. Shi, C. Wu, D. Tang, and H. Zhang "Recent progress of study on optical solitons in fiber lasers," Applied Physics Reviews, vol. 6, no. 2, 2019, doi: 10.1063/1.5091811.

[21] H. A. Haus, "Mode-locking of lasers," IEEE J. Sel. Top. Quantum Electron., vol. 6, no. 6, pp. 1173-1185, 2000, doi: $10.1109 / 2944.902165$.

[22] M. Singh and J. Malhotra, "40 Gbit/s-80 GHz hybrid MDM-OFDM-Multibeam based RoFSO transmission link under the effect of adverse weather conditions with enhanced detection," Optoelectronics and Advanced MaterialsRapid Communications, vol. 14, no. 3-4, pp. 146-153, 2019.

[23] D. A. J. Al-Khaffaf and I. A. Alshimaysawe, "Miniaturised tri-band microstrip patch antenna design for radio and millimetre waves of 5G devices," Indonesian Journal of Electrical Engineering and Computer Science, vol. 21, no. 3, pp1594-1601, 2021, doi: 10.11591/ijeecs.v21.i3.pp1594-1601.

[24] M. Singh and J. Malhotra, "A high-speed long-haul wavelength division multiplexing-based inter-satellite optical wireless communication link using spectral-efficient 2-D orthogonal modulation scheme," International Journal of Communication Systems, vol. 33, Article No: e4293, 2020, doi: 10.1002/dac.4293.

[25] M. Stratmann and F. Mitschke, "Chains of temporal dark solitons in dispersion-managed fiber," Phys. Rev. E, vol. 72, no. 6, 2005, doi: 10.1103/PhysRevE.72.066616. 
[26] S. M. Ao and J. R. Yan, "Effect of higher-order terms on nonlinear Schrodinger dark solitons in optical fibres," Chin. Phys. Lett., vol. 23, no. 10, pp. 2774-2777, 2006.

[27] S. Coen and T. Sylvestre, "Comment on "Dark pulse emission of a fiber laser," Phys. Rev. A, vol. 82, no. 4, 2010, doi: 10.1103/PhysRevA.82.047801.

[28] D. A. J. Al-Khaffaf and H. S. R. Hujijo, "High data rate optical wireless communication system using millimeter wave and optical phase modulation," ARPN Journal of Engineering and Applied Sciences, vol. 13, no. 23, pp. 9086-9092, dec. 2018.

[29] S. Manipatruni, et al., "Wide temperature range operation of micrometer-scale silicon electro-optic modulators," Optics Letters, vol. 33, no. 19, pp. 2185-2187, 2008, doi: 10.1364/OL.33.002185.

[30] B. G. Lee, A. Biberman, N. Sherwood-Droz, M. Lipson, and K. Bergman., "Thermally Active 4x4 Non-Blocking Switch for Networkson Chip,” in IEEE Lasers and Electro-Optics Society, pp. 370-371, 2008.

[31] K. Padmaraju, J. Chan, L. Chen, M. Lipson, and K. Bergman., "Thermal stabilization of a microring modulator using feedback control," Optics Express, vol. 20, no. 27, pp. 27999-28008, 2012, doi: 10.1364/OE.20.027999.

[32] Y. Ye, et al, "System-Level Modeling and Analysis of Thermal Effects in Optical Networks-on-Chip," IEEE Transactions on Very Large Scale Integration Systems, vol. 21, no. 2, pp. 292-305, February 2013, doi: 10.1109/TVLSI.2012.2185524.

[33] Z. Li, et al, "Reliability Modeling and Management of Nanophotonic On-Chip Networks," IEEE Transactions on Very Large Scale Integration (VLSI) Systems, vol. 20, no. 1, pp. 98-111, 2012, doi: 10.1109/TVLSI.2010.2089072.

[34] Z. Li, H. H. Tan, C. Jagadish, and L. Fu, "III-V Semiconductor Single Nanowire Solar Cells: A Review," Adv. Mater.Technol., vol. 3, no. 9, pp. 1800005, 2018, doi: 10.1002/admt.201800005.

[35] J. E. M. Haverkort, E. C. Garnett, and E. P. A. M. Bakkers, "Fundamentals of the nanowire solar cell: Optimizationof the open circuit voltage," Appl. Phys. Rev., vol. 5, no. 3, pp. 031106, 2018, doi: 10.1063/1.5028049.

[36] S. M. Frolov, S. R. Plissard, S. Nadj-Perge, L. P. Kouwenhoven, and E. P. A. M. Bakkers, "Quantum computingbased on semiconductor nanowires," MRS Bull., vol. 38, no. 10, pp. 809-815, 2013, doi: $10.1557 / \mathrm{mrs} .2013 .205$.

[37] X. Wu, et al., "Nanowire lasers as intracellular probes," Nanoscale, vol. 10, no. 20, pp. 9729-9735, 2018, doi: 10.1039/C8NR00515J.

[38] S. Nekita, et al., "Face-Selective Crystal Growth of Hydrothermal Tungsten Oxide Nanowires for Sensing VolatileMolecules," ACS Appl. Nano Mater., vol. 3, no. 10, pp. 10252-10260, 2020, doi: 10.1021/acsanm.0c02194.

[39] R. Yan, D. Gargas, and P. Yang, "Nanowire photonics," Nat. Photonics, vol. 3, no. 10, pp. 569-576, 2009.

[40] M. Notomi, M. Takiguchi, S. Sergent, G. Zhang, and H. Sumikura, "Nanowire photonics toward wide wavelengthrange and subwavelength confinement [Invited]," Opt. Mater. Express, vol. 10, no. 10, pp. 2560-2596, 2020, doi: 10.1364/OME.401317.

[41] H.-G. Park, "Nanowire Photonics," J. Korean Phys. Soc., vol. 73, no. 2, pp. 218-226, 2018, doi: $10.3938 / \mathrm{jkps} .73 .218$.

[42] H. Sumikura, et al., "Mid-Infrared Lasing of Single Wurtzite InAs Nanowire," Nano Lett., vol. 19, no. 11, pp. 8059-8065, 2019, doi: 10.1021/acs.nanolett.9b03249.

[43] S. Chen, M. Yukimune, R. Fujiwara, F. Ishikawa, W. M. Chen, and I. A. Buyanova, "Near-Infrared Lasing at $1 \mu \mathrm{mfrom}$ a Dilute-Nitride-Based Multishell Nanowire," Nano Lett., vol. 19. no. 2, pp. 885-890, 2019, doi: 10.1021/acs.nanolett.8b04103.

[44] S. Sergent, et al., "Subliming GaN into Ordered Nanowire Arrays for Ultraviolet and Visible Nanophotonics," ACS Photonics, vol. 6, no. 12, pp. 3321-3330, 2019, doi: 10.1021/acsphotonics.9b01435.

[45] S. Skalsky, et al., "Heterostructure and Q-factor engineering for low-threshold and persistent nanowire lasing," Light: Sci. Appl., vol. 9, no. 1, p. 43, 2020.

[46] D. Jevtics, et al., "Characterization, Selection, and Microassembly of Nanowire Laser Systems," Nano Lett., vol. 20, no. 3, pp. 1862-1868, 2020, doi: 10.1021/acs.nanolett.9b05078.

[47] J. Wang, et al., "High-efficiencybroadband second harmonic generation in single hexagonal GaAs nanowire," Sci. Rep., vol. 7, no. 1, p. 2166, 2017, doi: 10.1038/s41598-017-02199-w.

[48] Q. Yuan, et al., "Low-Power Continuous-WaveSecond Harmonic Generation in Semiconductor Nanowires," Laser and Photonics Reviews, vol. 12, no. 10, pp. 1800126, 2018, doi: 10.1002/lpor.201800126.

[49] G. Zhang, M. Takiguchi, K. Tateno, T. Tawara, M. Notomi, and H. Gotoh, "Telecom-band lasing in single InP/InAsheterostructure nanowires at room temperature," Sci. Adv., vol. 5, no. 2, 2019, doi: 10.1002/lpor.201800126. 